\title{
自然科学基金项目进展专栏
}

\section{解一维双曲守恒律方程和抛物方程的间断 有限元法的逐点和区间平均值误差估计}

献给林群教授 80 华诞

\author{
曹外香 ${ }^{1}$, 张智民 ${ }^{112}$ * \\ (1) 北京计算科学研究中心, 北京 100094 ; \\ (2) Department of Mathematics, Wayne State University, Detroit, MI 48202, USA \\ E-mail: wxcao@csrc.ac.cn, zzhang@math.wayne.edu
}

收稿日期: 2014-10-12；接受日期: 2015-03-18；＊通信作者

国家自然科学基金 (批准号: 11471031 和 91430216) 资助项目

\begin{abstract}
摘要 本文研究求解一维双曲守恒律方程和抛物方程的间断有限元法的超收敛性质. 具体来说, 对于 双曲守恒律方程和抛物方程, 当分别选择迎风和交替的数值流量时, 本文证明在合适的初始化条件下, 间断有限元解在迎风点上 (双曲方程) 或数值迹在节点上 (抛物方程) 的逐点误差和区间平均值误差均 以 $2 k+1$ 阶的速度收玫, 其中 $k$ 是间断有限元空间多项式的次数. 这个结果是对 Cao 等人 (2014) 以 及 Cao 和 Zhang (2014) 的超收敛结果的进一步改进和完善, 即把逐点误差的收敛阶由原来的 $2 k+\frac{1}{2}$ 提高到 $2 k+1$, 而区间平均值误差的收敛阶则从 $2 k$ 提高到 $2 k+1$, 从而与 $\mathrm{CaO}$ 等人 (2014)、Cao 和 Zhang (2014) 以及 Yang 和 Shu (2012) 的数值试验结果完全吻合.
\end{abstract}

关键词 超收敛间断有限元法 双曲方程 抛物方程 区间平均值 逐点误差估计

MSC (2010) 主题分类 $65 \mathrm{M} 15,65 \mathrm{M} 60,65 \mathrm{~N} 30$

\section{1 引言}

间断有限元或间断 Galerkin (discontinuous Galerkin, DG) 方法是一类使用完全不连续的分片多 项式空间作为解空间和检验函数空间的有限元方法. 它最早是 1973 年 Reed 和 Hill ${ }^{[1]}$ 在研究中子 输运方程时提出来的. 随后, Cockburn 和 Shu 采用间断有限元方法进行空间离散去求解与时间有关 的非线性方程 (参见文献 [2-6]), 进一步推动了这个方法的发展. 局部间断有限元 (LDG) 方法是间断 有限元法的一个延伸, 它主要用于求解各种含高阶导数的方程 (由于数值逼近在区间边界上的不连续 性, 间断有限元法不便于直接求解高阶方程). 局部间断有限元方法的基本思想就是把含高阶导数的 方程写成只含一阶导数的方程组形式, 然后再用间断有限元方法一一求解. 为了确保数值格式的稳定 性, 选取合适的数值流量是至关重要的. Cockburn 和 Shu ${ }^{[7]}$ 首次用局部间断有限元方法处理了与时 间有关的对流扩散方程. 此后, 局部间断有限元方法被应用到各种各样的高阶方程, 如含三阶导数的 Korteweg-de Vries (KdV) 方程 (参见文献 [8])、含时间项的双调和方程、完全非线性的 $k(n, n, n)$ 方程 和 Cahn-Hilliard 类型的方程等 (参见文献 [9-12]). 
间断有限元法解偏微分方程的超收玫性质也是近年来学者们比较感兴趣的研究领域, 这方面的内 容可参见文献 [13-20]. 最近, Yang 和 Shu ${ }^{[21]}$ 在研究一维双曲守恒律方程的超收敛性质时证明了 DG 数值逼近在右 Radau 点上的误差和单元平均值误差均以 $k+2$ 的阶数收玫; 同时他们也观测到 DG 数值解在迎风点上和单元平均值误差以 $2 k+1$ 的阶数收玫. 对此, 我们在文献 [22] 中重新研究了文 献 [21] 的数值结果所揭示的超收玫现象, 通过构造校正函数的方法, 给出了 DG 数值解在迎风点上 $2 k+1$ (平均意义下) 或 $2 k+\frac{1}{2}$ (逐点估计) 的严格数学证明. 此外, 我们还首次证明了 DG 数值解的 导数在左 Radau 点上以 $k+1$ 的超收玫阶逼近真解的导数. 与此同时, 我们还在文献 [23] 中证明了类 似的结果对抛物方程同样成立, 当然, 其证明过程较之守恒律方程要复杂得多 (由于采用 LDG 而产生 两个未知函数的影响). 在文献 $[22,23]$ 中, 我们虽然对求解双曲守恒律方程和抛物方程的 DG 方法的 超收玫性质有了比较完整的研究, 但是还遗留了两个问题, 一个是节点误差的逐点估计, 另外一个是区 间平均值误差估计. 理论上, 我们只能分别证明 $2 k+\frac{1}{2}$ 和 $2 k$ 阶, 然而数值算例却表明, 这两者的误差 收敛阶均能达到 $2 k+1$.

本文的主要目的就是要解决上述两个问题, 即我们将给出逐点和区间平均值误差 $2 k+1$ 收玫阶 的严格数学证明, 以此填补理论证明与数值算例之间的差距. 这里采用了不同于文献 $[22,23]$ 的证明 方法: 代之以建立数值解 $u_{h}$ 与真解的某个特殊插值函数 $u_{I}$ 的超逼近关系, 本文将证明 $u_{h}$ 与 $u_{I}$ 关 于时间求一次导数之后的超逼近结果, 即建立 $\left\|\left(u_{h}-u_{I}\right)_{t}\right\|_{0}$ 的超逼近关系从而得到间断有限元解的 逐点和单元平均值误差 $2 k+1$ 收玫阶的数学证明.

本文的结构如下: 第 2 节首先介绍一维双曲守恒律方程的间断有限元格式, 然后讨论校正函数的 构造过程和性质. 通过校正函数的作用, 我们给出了 DG 数值逼近在迎风点上的逐点误差以及区间平 均值误差 $2 k+1$ 收玫阶的严格数学证明. 第 3 节研究求解一维抛物方程的局部间断有限元格式以及 相应的超收玫性质. 我们证明了间断有限元逼近的数值迹在网格节点上的逐点误差以及区间平均值 误差均以 $2 k+1$ 阶的速度收玫. 为了避免重复, 本文将不再提供数值例子. 有兴趣的读者请参见文 献 [21-23] 中相应的数值实验结果.

若无特别说明, 本文中所出现的符号 “ $A \lesssim B$ ” 均表示 $A$ 可以被 $B$ 乘以一个常数 $C$ 所控制, 其 中常数 $C$ 不依赖于网格长度 $h$. 对任意的正整数 $m$ 和 $p$, 我们用 $W^{m, p}(D)$ 表示子区域 $D \subset \Omega$ 上标 准的 Soblev 空间, 其中范数和半范数分别用 $\|\cdot\|_{m, p, D}$ 和 $|\cdot|_{m, p, D}$ 表示. 当 $D$ 为我们考虑的整个区 域 $\Omega$ 时, $\|\cdot\|_{m, p, D}$ 和 $|\cdot|_{m, p, D}$ 分别简记为 $\|\cdot\|_{m, p}$ 和 $|\cdot|_{m, p}$. 当 $p=2$ 时, 我们记 $W^{m, p}(D)=H^{m}(D)$, $\|\cdot\|_{m, p, D}=\|\cdot\|_{m, D},|\cdot|_{m, p, D}=|\cdot|_{m, D}$.

\section{2 双曲守恒律方程}

不失一般性, 我们考虑如下双曲守恒律方程:

$$
\begin{aligned}
& u_{t}+u_{x}=0, \quad(x, t) \in[0,2 \pi] \times(0, T], \\
& u(x, 0)=u_{0}(x), \quad x \in \mathbb{R},
\end{aligned}
$$

这里假设初值 $u_{0}$ 充分光滑. 我们将研究两种边界条件: 周期边界条件 $u(0, t)=u(2 \pi, t)$ 和 Dirichlet 边 界条件 $u(0, t)=g(t)$.

\section{1 间断有限元格式}

对区域 $\Omega=[0,2 \pi]$ 作剖分, 节点为 $0=x_{\frac{1}{2}}<x_{\frac{3}{2}}<\cdots<x_{N+\frac{1}{2}}=2 \pi$. 对任意的正整数 $r$, 记 
$\mathbb{Z}_{r}=\{1, \ldots, r\}$, 并令 $\tau_{j}=\left(x_{j-\frac{1}{2}}, x_{j+\frac{1}{2}}\right), x_{j}=\frac{1}{2}\left(x_{j-\frac{1}{2}}+x_{j+\frac{1}{2}}\right), j \in \mathbb{Z}_{N}$ 分别表示区间单元和单元中点. 记 $h_{j}=x_{j+\frac{1}{2}}-x_{j-\frac{1}{2}}, \bar{h}_{j}=h_{j} / 2$ 以及 $h=\max _{j} h_{j}$, 并假设剖分是正则的, 即存在常数 $c$ 使得

$$
h \leqslant c h_{j}, \quad j \in \mathbb{Z}_{N} .
$$

取有限元空间 $V_{h}$ 为相应于剖分的分片 $k(k \geqslant 1)$ 次多项式空间, 即

$$
V_{h}=\left\{v:\left.v\right|_{\tau_{j}} \in \mathbb{P}_{k}\left(\tau_{j}\right), j \in \mathbb{Z}_{N}\right\},
$$

其中 $\mathbb{P}_{k}$ 表示次数不大于 $k$ 的多项式的集合.

解方程 (2.1) 的间断有限元法为, 寻找 $u_{h} \in V_{h}$ 使得对任意的 $v \in V_{h}$,

$$
\left(u_{h t}, v\right)_{j}-\left(u_{h}, v_{x}\right)_{j}+\left.\hat{u}_{h}\right|_{j+\frac{1}{2}} v_{j+\frac{1}{2}}^{-}-\left.\hat{u}_{h}\right|_{j-\frac{1}{2}} v_{j-\frac{1}{2}}^{+}=0,
$$

其中 $\left(u_{h t}, v\right)_{j}=\int_{\tau_{j}} u_{h t} v d x, v_{j+\frac{1}{2}}^{-}$和 $v_{j+\frac{1}{2}}^{+}$分别表示函数 $v$ 在点 $x_{j+\frac{1}{2}}$ 的左右极限; $\hat{u}_{h}$ 表示数值流量, 即数值解在单元边界处的取值, 该值往往依赖于不连续的数值解在单元两边的函数值. 本文采用迎风 的流量, 即

$$
\left.\hat{u}_{h}\right|_{j+\frac{1}{2}}=u_{h}\left(x_{j+\frac{1}{2}}^{-}\right), \quad j \in \mathbb{Z}_{N} .
$$

对于周期边界条件, 我们取边界点 $x=x_{\frac{1}{2}}$ 上的数值流量为

$$
\left.\hat{u}_{h}\right|_{\frac{1}{2}}=u_{h}\left(x_{N+\frac{1}{2}}^{-}\right),
$$

而对于 Dirichlet 边界条件, 我们则取

$$
\left.\hat{u}_{h}\right|_{\frac{1}{2}}=g .
$$

为了分析的方便, 我们仍记 $\left.\hat{u}_{h}\right|_{\frac{1}{2}}=u_{h}\left(x_{\frac{1}{2}}^{-}\right)$.

定义空间

$$
H_{h}^{1}=\left\{v:\left.v\right|_{\tau_{j}} \in H^{1}\left(\tau_{j}\right), j \in \mathbb{Z}_{N}\right\} .
$$

对任意的 $\varepsilon, v \in H_{h}^{1}$, 我们定义双线性形式

$$
a(\varepsilon, v)=\sum_{j=1}^{N} a_{j}(\varepsilon, v)
$$

其中

$$
a_{j}(\varepsilon, v)=\left(\varepsilon_{t}, v\right)_{j}-\left(\varepsilon, v_{x}\right)_{j}+\varepsilon_{j+\frac{1}{2}}^{-} v_{j+\frac{1}{2}}^{-}-\varepsilon_{j-\frac{1}{2}}^{-} v_{j-\frac{1}{2}}^{+} .
$$

显然, 我们从 $(2.2)$ 可得

$$
a\left(u_{h}, v\right)=0, \quad v \in V_{h} .
$$

同样地, 真解 $u$ 也满足 $(2.2)$, 从而,

$$
a(u, v)=0, \quad v \in V_{h} .
$$

此外, 当 $v \in H_{h}^{1}$ 满足条件 $v_{\frac{1}{2}}^{-}=0$ 或者 $v_{\frac{1}{2}}^{-}=v_{N+\frac{1}{2}}^{-}$时,

$$
a(v, v)=\left(v_{t}, v\right)+\frac{1}{2} \sum_{j=1}^{N}\left(v_{j+\frac{1}{2}}^{-} v_{j+\frac{1}{2}}^{-}+v_{j-\frac{1}{2}}^{+} v_{j-\frac{1}{2}}^{+}-2 v_{j-\frac{1}{2}}^{-} v_{j-\frac{1}{2}}^{+}\right)
$$




$$
\begin{aligned}
& =\left(v_{t}, v\right)+\frac{1}{2} \sum_{j=1}^{N}[v]_{j-\frac{1}{2}}^{2}+\frac{1}{2}\left(v_{N+\frac{1}{2}}^{-} v_{N+\frac{1}{2}}^{-}-v_{\frac{1}{2}}^{-} v_{\frac{1}{2}}^{-}\right) \\
& \geqslant\left(v_{t}, v\right),
\end{aligned}
$$

其中 $[v]_{j-\frac{1}{2}}=v_{j-\frac{1}{2}}^{+}-v_{j-\frac{1}{2}}^{-}$表示函数 $v$ 在点 $x_{j-\frac{1}{2}}$ 上的跳跃. 从而, 在这两种条件下, 我们都有

$$
\frac{1}{2} \frac{d}{d t}\|v\|_{0}^{2}=\left(v_{t}, v\right) \leqslant a(v, v)
$$

\section{2 分析}

给定 $v \in H_{h}^{1}$, 我们首先定义 $v$ 的 Gauss-Radau 投影 $P_{h}^{-} v \in V_{h}$ 如下:

$$
\left(P_{h}^{-} v, \varepsilon\right)_{j}=(v, \varepsilon)_{j}, \quad \forall \varepsilon \in \mathbb{P}_{k-1}\left(\tau_{j}\right), \quad P_{h}^{-} v\left(x_{j+\frac{1}{2}}^{-}\right)=v\left(x_{j+\frac{1}{2}}^{-}\right) .
$$

注意到算子 $P_{h}^{-}$在间断有限元法的误差分析中经常被使用, 如文献 $[21,24]$.

为了证明 $2 k+1$ 阶的强超收玫结果, 我们采取与传统间断有限元理论分析不同的技术路线, 使用 有限元超收玫理论中的超逼近手段 (参见文献 [25-30]). 具体来说就是构造一个特殊的插值函数使其 以 “尽可能高” 的阶逼近数值解 $u_{h}$. 对于一般的超收玫结果, 只要比全局最佳收玫阶高出一阶就可以, 而这里则要求高出 $k$ 阶, 传统的方式是无法满足要求的. 为了达到更高阶的超逼近, 我们对 Radau 投 影构造校正函数, 即令特殊的插值函数 $u_{I}=P_{h}^{-} u-w \in V_{h}$, 其中 $w$ 是待求的校正函数. 我们的目标 是使得 $u_{I}$ 以 $2 k+1$ 阶的速度超逼近 $u_{h}$ 并且在节点上与 $P_{h}^{-} u$ 保持一致, 这样就可以证明 $u_{h}$ 在节点 的迎风方向以 $2 k+1$ 阶的速度收玫于 $u$. 需要指出的是, 这里的校正函数与文献 [25] 中有限元法所使 用的校正函数的不同之处在于, 我们采用的是构造性而非存在性证明, 即对每一个 $k$, 我们给出 $w$ 的 显式表达, 这一点在数值试验中尤其重要. 因为我们证明的是半离散的结果, 而数值试验却必须做全离 散, 显式构造对于初值的处理至关重要, 关于这一点详情参见文献 [22,23].

清楚起见, 我们具体描述一下思路和校正函数的构造过程. 若简单令 $u_{I}=P_{h}^{-} u$ 不作校正, 那么, 在 (2.3) 中取 $v=u_{h}-P_{h}^{-} u$ 并利用正交性质以及周期或 Dirichlet 边界条件可得

$$
\frac{1}{2} \frac{d}{d t}\left\|u_{h}-P_{h}^{-} u\right\|_{0}^{2} \lesssim a\left(u-P_{h}^{-} u, u_{h}-P_{h}^{-} u\right) .
$$

而对于任意的 $v \in V_{h}$, 由函数的逼近论知识可得

$$
a\left(u-P_{h}^{-} u, v\right) \lesssim h^{k+1}, \quad \forall v \in V_{h} .
$$

最终, 我们只能得到

$$
\left\|u_{h}-u_{I}\right\|_{0}=\left\|u_{h}-P_{h}^{-} u\right\|_{0}=O\left(h^{k+1}\right) .
$$

这与我们想要的超收玫目标相差甚远. 为此, 我们需要构造合适的插值函数 $u_{I}$ (或校正函数 $w$ ) 使得 $u_{I}$ 既具有 $P_{h}^{-} u$ 的某些性质, 如

$$
u_{I}\left(x_{j+\frac{1}{2}}^{-}\right)=u\left(x_{j+\frac{1}{2}}^{-}\right), \quad \forall j \in \mathbb{Z}_{N},
$$

同时又与数值解 $u_{h}$ 是超逼近的, 即存在某些正数 $l>0$ 使得

$$
\left\|u_{I}-u_{h}\right\|_{0}=O\left(h^{k+l+1}\right)
$$


或者

$$
a\left(u-u_{I}, v\right) \lesssim h^{k+l+1}, \quad \forall v \in V_{h} .
$$

任意给定正整数 $l$, 其中 $1 \leqslant l \leqslant k$, 我们在文献 [22] 中给出了校正函数 $w=w^{l}$ 的构造性证明. 具 体来说, 若设函数 $u-P_{h}^{-} u$ 在任意的区间 $\tau_{j}, j \in \mathbb{Z}_{N}$ 上有如下的 Legendre 展开式:

$$
\left(u-P_{h}^{-} u\right)(x, t)=\sum_{p=0}^{\infty} u_{j, p}(t) L_{j, p}(x),
$$

其中 $L_{j, p}$ 表示区间 $\tau_{j}$ 上的 $p$ 次 Legendre 多项式,

$$
u_{j, p}=\frac{2 p+1}{h_{j}}\left(u-P_{h}^{-} u, L_{j, p}\right)_{j}, \quad 0 \leqslant p \leqslant k, \quad u_{j, p}=\frac{2 p+1}{h_{j}}\left(u, L_{j, p}\right)_{j}, \quad p \geqslant k+1,
$$

那么, 由 $P_{h}^{-}$的性质可知, $u_{j, p}=0, p \leqslant k-1$, 从而, 由 Legendre 多项式的正交性质可得

$$
\left(u-P_{h}^{-} u, v\right)_{j}=u_{j, k}\left(L_{j, k}, v\right)_{j}, \quad \forall v \in V_{h} .
$$

记 $G_{i}(t)=\partial_{t}^{i} u_{j, k}(t)$ 并定义函数 $F_{i}(x), 1 \leqslant i \leqslant k$ 使其满足

$$
F_{i}\left(x_{j+\frac{1}{2}}^{-}\right)=0, \quad \bar{h}_{j}\left(F_{i+1}, v_{x}\right)_{j}=\left(F_{i}, v\right)_{j}, \quad i \geqslant 1, \quad \bar{h}_{j}\left(F_{1}, v_{x}\right)_{j}=\left(L_{j, k}, v\right)_{j} .
$$

文献 [22] 证明了 $F_{i}(x)$ 是存在唯一的并给出了具体表达式. 我们构造校正函数如下:

$$
\left.w\right|_{\tau_{j}}:=w^{l}=\sum_{i=1}^{l} w_{i}, \quad w_{i}(x, t)=\left(\bar{h}_{j}\right)^{i} G_{i}(t) F_{i}(x) .
$$

校正函数 $w_{i}, i \in \mathbb{Z}_{l}$ 具有如下性质 (参见文献 $[22]$ ):

$$
w_{i}\left(x_{j+\frac{1}{2}}^{-}\right)=0, \quad w_{i} \perp \mathbb{P}_{k-i-1}, \quad\left\|\partial_{t}^{r} w_{i}\right\|_{0, \infty} \lesssim h^{k+1+i}\left\|\partial_{t}^{r} u\right\|_{k+i+1, \infty}, \quad r=0,1,2 .
$$

经简单计算, 我们不难得到

$$
\left(w_{i t}, v\right)_{j}=\left(w_{i+1}, v_{x}\right)_{j}, \quad i \geqslant 1, \quad\left(u_{t}-P_{h}^{-} u_{t}, v\right)_{j}=\left(w_{1}, v_{x}\right)_{j},
$$

从而,

$$
\begin{aligned}
& a_{j}\left(w^{l}, v\right)=\left(\left(w^{l}\right)_{t}, v\right)_{j}-\left(w^{l}, v_{x}\right)_{j}=\left(w_{l t}, v\right)_{j}-\left(w_{1}, v_{x}\right), \\
& a_{j}\left(u-P_{h}^{-} u+w^{l}, v\right)=\left(u_{t}-P_{h}^{-} u_{t}, v\right)_{j}+\left(w_{l t}, v\right)_{j}-\left(w_{1}, v_{x}\right)=\left(w_{l t}, v\right)_{j} .
\end{aligned}
$$

定义 $u$ 的插值函数

$$
u_{I}=u_{I}^{l}=P_{h}^{-} u-w^{l}
$$

我们有如下结果 (参见文献 [22]).

引理 2.1 设 $u \in W^{k+l+2, \infty}(\Omega), 1 \leqslant l \leqslant k$ 是方程 $(2.1)$ 的解, 那么,

$$
\left|a_{j}\left(u-u_{I}^{l}, v\right)\right| \lesssim h^{k+l+1}\|u\|_{k+l+2, \infty, \tau_{j}}\|v\|_{0,1, \tau_{j}}, \quad \forall v \in V_{h} .
$$

若 $u_{h}$ 是方程 $(2.2)$ 的解且初值条件满足

$$
\left\|\left(u_{h}-u_{I}^{l}\right)(x, 0)\right\|_{0} \lesssim h^{k+l+1}\|u\|_{k+l+2, \infty},
$$


那么, 对于周期和 Dirichlet 边界条件均有

$$
\left\|u_{I}^{l}-u_{h}\right\|_{0}(t) \lesssim(1+t) h^{k+l+1}\|u\|_{k+l+2, \infty} .
$$

特别地, 如果我们取初值 $u_{h}(x, 0)=u_{I}^{l}(x, 0)$, 则有

$$
\left\|u_{I}^{l}-u_{h}\right\|_{0}(t) \lesssim t h^{k+l+1}\|u\|_{k+l+2, \infty} .
$$

\section{3 超收敛}

给定函数 $v$, 记 $\|v\|_{c}$ 为函数 $v$ 的区间平均值误差, 即

$$
\|v\|_{c}=\left(\frac{1}{N} \sum_{j=1}^{N}\left(\frac{1}{h_{j}} \int_{\tau_{j}} v d x\right)^{2}\right)^{\frac{1}{2}} .
$$

我们有如下关于函数 $u-u_{h}$ 的区间平均值误差的超收玫结果.

定理 2.2 设 $u \in W^{2 k+2, \infty}(\Omega)$ 和 $u_{h}$ 分别是方程 $(2.1)$ 和 $(2.2)$ 的解. 若选取特殊的初值 $u_{h}(\cdot, 0)$ 使得 (2.9) 对 $l=k$ 成立, 则对周期和 Direchlet 边界条件均有

$$
\left\|u-u_{h}\right\|_{c} \lesssim(1+t) h^{2 k+1}\|u\|_{2 k+2, \infty} .
$$

证明 取校正函数 $w=w^{k}$, 由 $P_{h}^{-} u$ 的性质可得

$$
\int_{\tau_{j}}\left(u-u_{I}^{k}\right)(x, t)=\int_{\tau_{j}}\left(u-P_{h}^{-} u+w^{k}\right)(x, t)=\int_{\tau_{j}} w^{k}(x, t)=\int_{\tau_{j}} w_{k}(x, t) d x,
$$

其中最后一步用到了 $(2.5)$ 第二式. 从而,

$$
\int_{\tau_{j}}\left(u-u_{h}\right)(x, t) d x=\int_{\tau_{j}}\left(u_{I}^{k}-u_{h}\right)(x, t) d x+\int_{\tau_{j}} w_{k}(x, t) d x .
$$

由 Cauchy 不等式和 (2.5) 第三式, 我们可得

$$
\left|\int_{\tau_{j}}\left(u-u_{h}\right)(x, t) d x\right| \lesssim h^{\frac{1}{2}}\left\|u_{I}^{k}-u_{h}\right\|_{0, \tau_{j}}+h^{2 k+2}\|u\|_{2 k+2, \infty} .
$$

经直接计算并在 $(2.10)$ 中取 $l=k$ 可得

$$
\left\|u-u_{h}\right\|_{c} \lesssim\left\|u_{I}^{k}-u_{h}\right\|_{0}+h^{2 k+1}\|u\|_{2 k+2, \infty} \lesssim(1+t) h^{2 k+1}\|u\|_{2 k+2, \infty} .
$$

从而, 定理得证.

接下来讨论数值解 $u_{h}$ 在迎风点上的逐点估计. 在文献 [22] 中, 我们曾利用 $u_{h}$ 与 $u_{I}^{k}$ 之间的超逼 近结果, 以此证明了 $u_{h}$ 在迎风点上的逐点误差 $2 k+\frac{1}{2}$ 的超收玫阶, 即

$$
\left|\left(u_{h}-u\right)_{j+\frac{1}{2}}^{-}\right|=\left|\left(u_{h}-u_{I}^{k}\right)_{j+\frac{1}{2}}^{-}\right| \lesssim\left\|u_{h}-u_{I}^{k}\right\|_{0, \infty} \lesssim h^{-\frac{1}{2}}\left\|u_{h}-u_{I}^{k}\right\|_{0} \lesssim(1+t) h^{2 k+\frac{1}{2}}\|u\|_{2 k+2, \infty} .
$$

注意到在上述证明中, 我们用逆不等式损失了 $1 / 2$ 阶, 从而, 逐点误差的收玫阶相应损失 $1 / 2$ 阶, 未能 达到最优收玫阶 $2 k+1$. 为了达到 $2 k+1$ 阶的目的, 本文将采用不同于文献 [22] 的证明方法. 我们的 证明思路是首先建立 $u_{I}^{k}$ 和 $u_{h}$ 对时间求一次导数后的强超逼近结果, 进而利用 $\left(u_{h}-u_{I}^{k}\right)_{t}$ 的超逼近 结果得到逐点误差 $2 k+1$ 收敛阶的严格数学证明. 
引理 2.3 设 $u \in W^{k+l+3, \infty}(\Omega), 1 \leqslant l \leqslant k$ 和 $u_{h}$ 分别是方程 $(2.1)$ 和 (2.2) 的解. 若初值 $u_{h}(x, 0)=u_{I}^{l}(x, 0)$, 则有

$$
\left\|\left(u_{I}^{l}-u_{h}\right)_{t}\right\|_{0}(t) \lesssim(1+t) h^{k+l+1}\|u\|_{k+l+3, \infty}, \quad \forall t \geqslant 0 .
$$

证明 由于 $u$ 和 $u_{h}$ 均满足方程 (2.2), 在方程两边同时关于 $t$ 求导可得

$$
a_{j}\left(u_{t}-u_{h t}, v\right)=0, \quad \forall v \in V_{h} .
$$

另一方面, 由校正函数的构造可知, $w_{t}^{l}\left(x_{j+\frac{1}{2}}^{-}, t\right)=0, \forall t \geqslant 0$, 所以, 对于周期边界条件成立着

$$
\left(u_{h}-u_{I}^{l}\right)_{t}\left(x_{\frac{1}{2}}^{-}, t\right)=\left(u_{h}-u_{I}^{l}\right)_{t}\left(x_{N+\frac{1}{2}}^{-}, t\right),
$$

而对于 Direchlet 边界条件则有

$$
\left(u_{h}-u_{I}^{l}\right)_{t}\left(x_{\frac{1}{2}}^{-}, t\right)=\left(u_{h}-P_{h}^{-} u\right)_{t}\left(x_{\frac{1}{2}}^{-}, t\right)=0 .
$$

从而, 在 (2.3) 中取 $v=\left(u_{h}-u_{I}^{l}\right)_{t}$ 并利用周期或 Direchlet 边界条件可得

$$
\frac{1}{2} \frac{d}{d t}\left\|\left(u_{h}-u_{I}^{l}\right)_{t}\right\|_{0}^{2} \leqslant a\left(\left(u_{h}-u_{I}^{l}\right)_{t},\left(u_{h}-u_{I}^{l}\right)_{t}\right)=a\left(\left(u-u_{I}^{l}\right)_{t},\left(u_{h}-u_{I}^{l}\right)_{t}\right) .
$$

对任意的 $v \in V_{h}$, 我们由 (2.5)-(2.7) 可得

$$
\left|a\left(\left(u-u_{I}^{l}\right)_{t}, v\right)\right|=\left|\left(\left(w_{l}\right)_{t t}, v\right)\right| \lesssim h^{k+l+1}\left\|\partial_{t}^{2} u\right\|_{k+l+1, \infty}\|v\|_{0,1} .
$$

注意到 $u_{t}=-u_{x}$ 和 $\|\cdot\|_{0,1} \lesssim\|\cdot\|_{0}$, 我们可得

$$
\frac{d}{d t}\left\|\left(u_{h}-u_{I}^{l}\right)_{t}\right\|_{0} \lesssim h^{k+l+1}\|u\|_{k+l+3, \infty} .
$$

从而, 对任意的 $t>0$,

$$
\left\|\left(u_{h}-u_{I}^{l}\right)_{t}\right\|_{0}(t)=\left\|\left(u_{h}-u_{I}^{l}\right)_{t}\right\|_{0}(0)+\int_{0}^{t} \frac{d}{d t}\left\|\left(u_{h}-u_{I}^{l}\right)_{t}\right\|_{0} \lesssim\left\|\left(u_{h}-u_{I}^{l}\right)_{t}\right\|_{0}(0)+t h^{k+l+1}\|u\|_{k+l+3, \infty} .
$$

接下来估计 $\left\|\left(u_{h}-u_{I}^{l}\right)_{t}\right\|_{0}(0)$. 在 $t=0$ 时刻, 因为 $u_{h}(x, 0)=u_{I}^{l}(x, 0)$, 所以, 由间断有限元格式可得

$$
0=a\left(e_{t}, v\right)(0)=a\left(\left(u-u_{I}^{l}\right)_{t}, v\right)(0)+a\left(\left(u_{I}^{l}-u_{h}\right)_{t}, v\right)(0)=a\left(\left(u-u_{I}^{l}\right)_{t}, v\right)(0)+\left(\left(u_{I}^{l}-u_{h}\right)_{t}, v\right)(0) .
$$

特别地, 若在上式中取 $v=\left(u_{I}^{l}-u_{h}\right)_{t}$ 并运用估计式 (2.14) 即可推出

$$
\left\|\left(u_{I}^{l}-u_{h}\right)_{t}\right\|_{0}(0) \lesssim h^{k+l+1}\|u\|_{k+l+3, \infty}
$$

从而, 引理得证.

定理 2.4 设 $u \in W^{2 k+3, \infty}(\Omega)$ 和 $u_{h}$ 分别是方程 (2.1) 和 $(2.2)$ 的解. 若 $u_{h}(x, 0)=u_{I}^{k}(x, 0)$, 则 对于周期和 Direchlet 边界条件均有

$$
\left|\left(u-u_{h}\right)\left(x_{j+\frac{1}{2}}^{-}, t\right)\right| \lesssim(1+t) h^{2 k+1}\|u\|_{2 k+3, \infty}, \quad \forall j \in \mathbb{Z}_{N} .
$$

证明 记

$$
e=u-u_{h}, \quad \xi=u_{I}^{k}-u_{h}=P_{h}^{-} u-w^{k}-u_{h}
$$


在方程 $a_{i}(e, v)=0$ 中取 $v=1$ 并利用 $P_{h}^{-}$的性质和 $(2.5)$ 第二式可得

$$
e_{i+\frac{1}{2}}^{-}-e_{i-\frac{1}{2}}^{-}=-\int_{\tau_{i}} e_{t}=-\int_{\tau_{i}}\left(\xi+w^{k}\right)_{t}=-\int_{\tau_{i}}\left(\xi+w_{k}\right)_{t} .
$$

把 $i$ 从 1 一直加到 $j, j \geqslant i$ 可得

$$
e_{j+\frac{1}{2}}^{-}-e_{\frac{1}{2}}^{-}=-\sum_{i=1}^{j} \int_{\tau_{i}}\left(\xi_{t}+w_{k t}\right)
$$

当边界取 Direclet 边界条件时, 因为 $e_{\frac{1}{2}}^{-}=0$, 所以, 我们从 (2.5) 第三式和 (2.13) 可推导出

$$
\left|e_{j+\frac{1}{2}}^{-}\right| \lesssim\left\|\xi_{t}\right\|_{0}+\left\|w_{k t}\right\|_{0, \infty} \lesssim(1+t) h^{2 k+1}\|u\|_{2 k+3, \infty} .
$$

从而, (2.15) 对于 Direchlet 边界条件成立.

当边界取周期边界条件时, 不妨假设函数 $\xi$ 在任意区间 $\tau_{j}, j \in \mathbb{Z}_{N}$ 上有如下表达式:

$$
\xi(x, t)=\xi\left(x_{j}, t\right)+s(x, t) \frac{x-x_{j}}{h_{j}}, \quad \forall x \in \tau_{j} .
$$

对任意的 $v \in V_{h}$, 若 $v_{j-\frac{1}{2}}^{+}=0$, 由正交性可得

$$
0=a_{j}(e, v)=a_{j}\left(u-u_{I}^{k}, v\right)+a_{j}(\xi, v)=a_{j}\left(u-u_{I}^{k}, v\right)+\left(\xi_{t}, v\right)_{j}+\left(\xi_{x}, v\right)_{j},
$$

其中在最后一步, 我们用到了分部积分公式和 $v_{j-\frac{1}{2}}^{+}=0$ 这个条件. 特别地, 若取 $v=s(x, t) \frac{x-x_{j-\frac{1}{2}}}{h_{j}}$, 显 然, $v_{j-\frac{1}{2}}^{+}=0$, 从而,

$$
\int_{\tau_{j}} s(x, t) \frac{x-x_{j-\frac{1}{2}}}{h_{j}} \frac{d}{d x}\left(s(x, t) \frac{x-x_{j}}{h_{j}}\right)=-a_{j}\left(u-u_{I}^{k}, v\right)-\left(\xi_{t}, v\right)_{j} .
$$

因为 (参见文献 $[24]$ )

$$
\int_{\tau_{j}} s(x) \frac{x-x_{j-\frac{1}{2}}}{h_{j}} \frac{d}{d x}\left(s(x) \frac{x-x_{j}}{h_{j}}\right)=\frac{1}{4 h_{j}} \int_{\tau_{j}} s^{2}(x)+\frac{s^{2}\left(x_{j+\frac{1}{2}}\right)}{4},
$$

所以, 由 (2.8) 和 (2.13) 可得

$$
\|s\|_{0, \tau_{j}}^{2} \lesssim h_{j}\left|a_{j}\left(u-u_{I}^{k}, v\right)+\left(\xi_{t}, v\right)_{j}\right| \lesssim(1+t) h^{2 k+2}\|u\|_{2 k+3, \infty}\|v\|_{0, \tau_{j}} .
$$

注意到 $\|v\|_{0, \tau_{j}} \lesssim\|s\|_{0, \tau_{j}}$, 我们有

$$
\|s\|_{0, \tau_{j}} \lesssim(1+t) h^{2 k+2}\|u\|_{2 k+3, \infty}, \quad\|s\|_{0, \infty, \tau_{j}} \lesssim h_{j}^{-\frac{1}{2}}\|s\|_{0, \tau_{j}} \lesssim(1+t) h^{2 k+\frac{3}{2}}\|u\|_{2 k+3, \infty} .
$$

另一方面, 在公式 $a(e, v)=0$ 中取 $v=1$ 并利用周期边界条件 $e_{N+\frac{1}{2}}^{-}=e_{\frac{1}{2}}^{-}=0$ 可得

$$
\frac{d}{d t} \int_{\Omega}\left(\xi+w^{k}\right)=\int_{\Omega}\left(\xi+w^{k}\right)_{t}=\int_{\Omega} e_{t}=0
$$

从而, 对任意的 $t \geqslant 0$,

$$
\int_{\Omega} \xi(x, t) d x=\int_{\Omega}\left(\xi+w^{k}\right)(x, 0) d x-\int_{\Omega} w^{k}(x, t) d x=\int_{\Omega}\left(w_{k}(x, 0)-w_{k}(x, t)\right) d x .
$$


把 $\xi$ 的表达式 (2.17) 代入上式可得

$$
\sum_{j=1}^{N}\left(h_{j} \xi\left(x_{j}, t\right)+\int_{\tau_{j}} s(x, t) \frac{x-x_{j}}{h_{j}}\right)=\sum_{j=1}^{N} \int_{\tau_{j}}\left(w_{k}(x, 0)-w_{k}(x, t)\right) d x .
$$

同理, 由于 $e_{j+\frac{1}{2}}^{-}=\xi_{j+\frac{1}{2}}^{-}$, 从而, 由 (2.16) 可知,

$$
\xi_{j+\frac{1}{2}}^{-}-\xi_{\frac{1}{2}}^{-}=e_{j+\frac{1}{2}}^{-}-e_{\frac{1}{2}}^{-}=-\sum_{i=1}^{j} \int_{\tau_{i}}\left(\xi+w_{k}\right)_{t}
$$

即

$$
\xi\left(x_{j}, t\right)=\xi\left(x_{1}, t\right)+\frac{1}{2} s\left(x_{\frac{1}{2}}^{-}, t\right)-\frac{1}{2} s\left(x_{j+\frac{1}{2}}^{-}, t\right)-\sum_{i=1}^{j} \int_{\tau_{i}}\left(\xi+w_{k}\right)_{t} .
$$

把上式代入 (2.18) 并利用不等式 (2.5) 和 (2.13) 可得

$$
\left|\xi\left(x_{1}, t\right)\right| \lesssim\|s\|_{0, \infty}+\left\|w_{k}\right\|_{0, \infty}+\left\|w_{k}\right\|_{0, \infty}(0)+\left\|w_{k t}\right\|_{0, \infty}+\left\|\xi_{t}\right\|_{0} \lesssim(1+t) h^{2 k+1}\|u\|_{2 k+3, \infty} .
$$

从而, 对任意的 $j \in \mathbb{Z}_{N}$,

$$
\left|\xi\left(x_{j}, t\right)\right| \lesssim\left|\xi\left(x_{1}, t\right)\right|+\|s\|_{0, \infty}+\left\|w_{k t}\right\|_{0, \infty}+\left\|\xi_{t}\right\|_{0} \lesssim(1+t) h^{2 k+1}\|u\|_{2 k+3, \infty} .
$$

所以,

$$
\left|\xi_{j+\frac{1}{2}}^{-}\right|=\left|\xi\left(x_{j}, t\right)+\frac{1}{2} s\left(x_{j+\frac{1}{2}}^{-}, t\right)\right| \lesssim(1+t) h^{2 k+1}\|u\|_{2 k+3, \infty} .
$$

从而, 不等式 (2.15) 对于周期边界条件同样也成立. 定理得证.

注 2.5 在上述定理中, 我们对解的正则性要求略高于文献 [22], 后者为 $u \in W^{2 k+2, \infty}(\Omega)$. 其主 要原因在于, 这里用到了 $\left\|\left(u_{h}-u_{I}^{k}\right)_{t}\right\|_{0}$ 的误差估计而在文献 [22] 中只用到 $\left\|u_{h}-u_{I}^{k}\right\|_{0}$ 的估计.

注 2.6 初值条件 $u_{h}(x, 0)=u_{I}^{k}(x, 0)=P_{h}^{-} u_{0}(x)-w^{k}(x, 0)$ 意味着我们需要对初始误差作校正. 事实上, 我们在文献 [22] 中曾指出, 若对初值不作校正, 例如, 初值直接取为真解的 $L^{2}$ 投影或 Radau 插值, 那么, 当 $k \geqslant 3$ 时, 我们在数值上观测不到间断有限元解在迎风点上 $2 k+1$ 的超收玫阶.

\section{3 一维抛物方程}

\section{1 局部间断有限元格式}

考虑如下模型问题:

$$
\begin{aligned}
& u_{t}=u_{x x}, \quad(x, t) \in[0,2 \pi] \times(0, T], \\
& u(x, 0)=u_{0}(x), \quad x \in \mathbb{R},
\end{aligned}
$$

这里仍假设 $u_{0}$ 充分光滑. 我们将研究三种不同的边界条件: 周期边界条件 $u(0, t)=u(2 \pi, t)$ 以及两种 混合边界条件 $u(0, t)=g_{0}(t), u_{x}(2 \pi, t)=g_{1}(t)$ 和 $u_{x}(0, t)=g_{0}(t), u(2 \pi, t)=g_{1}(t)$.

我们采用局部间断有限元法来求解方程 (3.1). 为了构造局部间断有限元格式, 首先把方程 $(3.1)$ 写成两个一阶方程组的形式:

$$
u_{t}=q_{x}, \quad q=u_{x}
$$


求解方程 (2.1) 的间断有限元法为, 寻找 $\left(u_{h}, q_{h}\right) \in V_{h}$ 使得对任意的 $v, \varphi \in V_{h}$,

$$
\begin{aligned}
& \left(u_{h t}, v\right)_{j}=-\left(q_{h}, v_{x}\right)_{j}+\left.\hat{q}_{h} v^{-}\right|_{j+\frac{1}{2}}-\left.\hat{q}_{h} v^{+}\right|_{j-\frac{1}{2}}, \\
& \left(q_{h}, \varphi\right)_{j}=-\left(u_{h}, \varphi_{x}\right)_{j}+\left.\hat{u}_{h} \varphi^{-}\right|_{j+\frac{1}{2}}-\left.\hat{u}_{h} \varphi^{+}\right|_{j-\frac{1}{2}},
\end{aligned}
$$

这里选取交替的数值流量, 即

$$
\hat{u}_{h}=u_{h}^{-}, \quad \hat{q}_{h}=q_{h}^{+},
$$

或者

$$
\hat{u}_{h}=u_{h}^{+}, \quad \hat{q}_{h}=q_{h}^{-} .
$$

当边界为周期边界条件时, 我们取流量 (3.4) 或 (3.5) 并且在边界上取

$$
\left.\hat{u}_{h}\right|_{\frac{1}{2}}=\left.\hat{u}_{h}\right|_{N+\frac{1}{2}},\left.\quad \hat{q}_{h}\right|_{\frac{1}{2}}=\left.\hat{q}_{h}\right|_{N+\frac{1}{2}} .
$$

当边界条件为 $u(0, t)=g_{0}(t), u_{x}(2 \pi, t)=g_{1}(t)$ 时, 我们选择流量 (3.4) 并且在边界点上取

$$
\left.\hat{u}_{h}\right|_{\frac{1}{2}}=\left.u_{h}^{-}\right|_{\frac{1}{2}}=g_{0},\left.\quad \hat{q}_{h}\right|_{N+\frac{1}{2}}=\left.q_{h}^{+}\right|_{N+\frac{1}{2}}=g_{1} .
$$

同样地, 当边界条件为 $u_{x}(0, t)=g_{0}(t), u(2 \pi, t)=g_{1}(t)$ 时, 我们选择流量 (3.5) 并且取边界上的流量

$$
\left.\hat{q}_{h}\right|_{\frac{1}{2}}=\left.q_{h}^{-}\right|_{\frac{1}{2}}=g_{0},\left.\quad \hat{u}_{h}\right|_{N+\frac{1}{2}}=\left.u_{h}^{+}\right|_{N+\frac{1}{2}}=g_{1} .
$$

对任意的 $\varepsilon, \eta, v \in H_{h}^{1}$, 我们定义

$$
a^{1}(\varepsilon, \eta ; v)=\sum_{j=1}^{N} a_{j}^{1}(\varepsilon, \eta ; v), \quad a^{2}(\varepsilon, \eta ; v)=\sum_{j=1}^{N} a_{j}^{2}(\varepsilon, \eta ; v),
$$

其中

$$
\begin{aligned}
& a_{j}^{1}(\varepsilon, \eta ; v)=\left(\varepsilon_{t}, v\right)_{j}+\left(\eta, v_{x}\right)_{j}-\left.\hat{\eta} v^{-}\right|_{j+\frac{1}{2}}+\left.\hat{\eta} v^{+}\right|_{j-\frac{1}{2}}, \\
& a_{j}^{2}(\varepsilon, \eta ; v)=(\eta, v)_{j}+\left(\varepsilon, v_{x}\right)_{j}-\left.\hat{\varepsilon} v^{-}\right|_{j+\frac{1}{2}}+\left.\hat{\varepsilon} v^{+}\right|_{j-\frac{1}{2}} .
\end{aligned}
$$

这里数值流量 $(\hat{\varepsilon}, \hat{\eta})$ 取 $(3.4)$ 或者 $(3.5)$. 由 (3.3) 我们可得

$$
a^{1}\left(u_{h}, q_{h} ; v\right)=0, \quad a^{2}\left(u_{h}, q_{h} ; \varphi\right)=0, \quad \forall v, \varphi \in V_{h} .
$$

由于真解 $(u, q)$ 也满足方程 $(3.3)$, 从而有

$$
a^{1}(u, q ; v)=0, \quad a^{2}(u, q ; \varphi)=0, \quad \forall v, \varphi \in V_{h} .
$$

当流量 $(\hat{\varepsilon}, \hat{\eta})$ 取 $(3.4)$ 时, 经直接计算可得

$$
a^{1}(\varepsilon, \eta ; \varepsilon)+a^{2}(\varepsilon, \eta ; \eta)=\left(\varepsilon_{t}, \varepsilon\right)+(\eta, \eta)-\left.\eta^{+} \varepsilon^{-}\right|_{N+\frac{1}{2}}+\left.\eta^{+} \varepsilon^{-}\right|_{\frac{1}{2}},
$$

而当 $(\hat{\varepsilon}, \hat{\eta})$ 取 $(3.5)$ 时, 则有

$$
a^{1}(\varepsilon, \eta ; \varepsilon)+a^{2}(\varepsilon, \eta ; \eta)=\left(\varepsilon_{t}, \varepsilon\right)+(\eta, \eta)-\left.\eta^{-} \varepsilon^{+}\right|_{N+\frac{1}{2}}+\left.\eta^{-} \varepsilon^{+}\right|_{\frac{1}{2}} .
$$




\section{2 分析}

有别于双曲守恒律方程, 在利用校正函数分析抛物方程的超收玫性质时, 由于两个变量的缘故 (真 解 $u$ 和辅助变量 $q=u_{x}$ ), 我们需要对两个变量同时校正. 此外, 因为变量是相关的, 所以, 变量的校正 函数也会互相影响, 而如何平衡校正函数的相互影响, 使得最终达到我们的超收玫目的, 是我们在构造 校正函数时需要特别注意的, 这也是抛物方程乃至高阶方程区别于双曲守恒律方程的本质所在.

对任意的函数 $v \in H_{h}^{1}$, 我们首先定义 $v$ 的另外一个 Gauss-Radau 投影算子 $P_{h}^{+} v$ 如下:

$$
\left(P_{h}^{+} v, \varepsilon\right)_{j}=(v, \varepsilon)_{j}, \quad \forall \varepsilon \in \mathbb{P}_{k-1}\left(\tau_{j}\right), \quad P_{h}^{+} v\left(x_{j-\frac{1}{2}}^{+}\right)=v\left(x_{j-\frac{1}{2}}^{+}\right) .
$$

对于抛物方程来说, 我们需要同时构造 $u$ 和 $q$ 的校正函数 $w_{u}$ 和 $w_{q}$ 使得真解的插值函数 $\left(u_{I}, q_{I}\right)$ $=\left(P_{h} u-w_{u}, P_{h} q-w_{q}\right)$ 超逼近数值解 $\left(u_{h}, p_{h}\right)$, 并且同时满足

$$
\hat{u}_{I}\left(x_{j+\frac{1}{2}}\right)=\hat{u}\left(x_{j+\frac{1}{2}}\right), \quad \hat{q}_{I}\left(x_{j+\frac{1}{2}}\right)=\hat{q}\left(x_{j+\frac{1}{2}}\right),
$$

其中 $P_{h}=P_{h}^{-}$或 $P_{h}^{+}$. 由 (3.6) 和 (3.7) 我们可得, 对 $\left\|u_{h}-u_{I}\right\|_{0}+\left\|q_{h}-q_{I}\right\|_{0}$ 的估计最终会转化到

$$
a^{1}\left(u-u_{I}, q-q^{I} ; u_{h}-u_{I}\right)+a^{2}\left(u-u_{I}, q-q^{I} ; q_{h}-q_{I}\right)
$$

的估计上去. 事实上, 无论是选择流量 (3.4) 或 (3.5), 在相应的边界条件下, 我们都可得到

$$
\left.\left(\hat{u}-\hat{u}_{h}\right)\left(\hat{q}-\hat{q}_{h}\right)\right|_{N+\frac{1}{2}}=\left.\left(\hat{u}-\hat{u}_{h}\right)\left(\hat{q}-\hat{q}_{h}\right)\right|_{\frac{1}{2}} .
$$

所以, 在 $(3.6)$ 或 $(3.7)$ 上取 $(\varepsilon, \eta)=\left(u_{h}-u_{I}, q_{h}-q_{I}\right)$ 即可得

$$
\begin{aligned}
\frac{1}{2} \frac{d}{d t}\left\|u_{h}-u_{I}\right\|_{0}^{2}+\left\|q_{h}-q_{I}\right\|_{0}^{2} & =a^{1}\left(u_{h}-u_{I}, q_{h}-q_{I} ; u_{h}-u_{I}\right)+a^{2}\left(u_{h}-u_{I}, q_{h}-q_{I} ; q_{h}-q_{I}\right) \\
& =a^{1}\left(u-u_{I}, q-q_{I} ; u_{h}-u_{I}\right)+a^{2}\left(u-u_{I}, q-q_{I} ; q_{h}-q_{I}\right),
\end{aligned}
$$

其中最后一步用到了正交性质. 因为对任意的 $v, \varphi \in V_{h}$,

$$
\begin{aligned}
& a^{1}\left(u-u_{I}, q-q_{I} ; v\right)=\left(u_{t}-P_{h} u_{t}, v\right)+\left(w_{u t}, v\right)+\left(w_{q}, v_{x}\right), \\
& a^{2}\left(u-u_{I}, q-q_{I} ; \varphi\right)=\left(q-P_{h} q, \varphi\right)+\left(w_{q}, \varphi\right)+\left(w_{u}, \varphi_{x}\right) .
\end{aligned}
$$

所以, 我们需要构造校正函数 $\left(w_{u}, w_{q}\right)$, 使得对任意的 $v, \varphi \in V_{h}$,

$$
a^{1}\left(u-u_{I}, q-q_{I} ; v\right) \lesssim h^{k+l+1}, \quad a^{2}\left(u-u_{I}, q-q_{I} ; \varphi\right) \lesssim h^{k+l+1},
$$

其中 $l$ 是某些大于 0 的正数.

文献 [23] 给出了校正函数的构造性证明并研究了它的一些性质. 在阐述校正函数的性质之前, 我 们还需要一些额外的记号. 首先, 对任意的正数 $r,\lfloor r\rfloor$ 和 $\lceil r\rceil$ 分别表示不大于 $r$ 的最大的整数和不小 于 $r$ 的最小整数. 其次, 记

$$
\begin{array}{ll}
\left(P_{h} u, P_{h} q\right)=\left(P_{h}^{-} u, P_{h}^{+} q\right), & \text { 若 }\left(\hat{u}_{h}, \hat{q}_{h}\right)=\left(u_{h}^{-}, q_{h}^{+}\right), \\
\left(P_{h} u, P_{h} q\right)=\left(P_{h}^{+} u, P_{h}^{-} q\right), & \text { 若 }\left(\hat{u}_{h}, \hat{q}_{h}\right)=\left(u_{h}^{+}, q_{h}^{-}\right) .
\end{array}
$$

引理 $3.1^{[23]}$ 给定正整数 $l$, 其中 $1 \leqslant l \leqslant k$, 存在校正函数

$$
w_{q}^{l}(x, t)=\sum_{i=1}^{\lceil l / 2\rceil} w_{q, i}+\sum_{i=1}^{\lfloor l / 2\rfloor} \bar{w}_{q, i}, \quad w_{u}^{l}(x, t)=\sum_{i=1}^{\lceil l / 2\rceil} w_{u, i}+\sum_{i=1}^{\lfloor l / 2\rfloor} \bar{w}_{u, i},
$$


满足如下条件: 当流量 $\left(\hat{u}_{h}, \hat{q}_{h}\right)$ 取 (3.4) 时,

$$
w_{u}^{l}\left(x_{j+\frac{1}{2}}^{-}\right)=w_{q}^{l}\left(x_{j-\frac{1}{2}}^{+}\right)=0 ;
$$

当流量取 (3.5) 时,

$$
w_{q}^{l}\left(x_{j+\frac{1}{2}}^{-}\right)=w_{u}^{l}\left(x_{j-\frac{1}{2}}^{+}\right)=0
$$

并且当 $l=2 r$ 为偶数时,

$$
a^{1}\left(u-u_{I}^{l}, q-q_{I}^{l} ; v\right)=\left(\left(\bar{w}_{u, r}\right)_{t}, v\right), \quad a^{2}\left(u-u_{I}^{l}, q-q_{I}^{l} ; v\right)=\left(\bar{w}_{q, r}, v\right) ;
$$

当 $l=2 r+1$ 为奇数时,

$$
a^{1}\left(u-u_{I}^{l}, q-q_{I}^{l} ; v\right)=\left(\left(w_{u, r+1}\right)_{t}, v\right), \quad a^{2}\left(u-u_{I}^{l}, q-q_{I}^{l} ; v\right)=\left(w_{q, r+1}, v\right) .
$$

此外, $w_{v, i}, \bar{w}_{v, i}, v=u, q$ 满足

$$
w_{v, i} \perp \mathbb{P}_{k-2 i}, \quad \bar{w}_{v, i} \perp \mathbb{P}_{k-2 i-1},
$$

并且当 $u \in W^{k+l+2, \infty}(\Omega), 1 \leqslant l \leqslant k$ 时,

$$
\begin{aligned}
\left\|w_{q, i}\right\|_{0, \infty, \tau_{j}} & \lesssim h^{k+2 i}\|u\|_{k+1+2 i, \infty, \tau_{j}}, \quad\left\|\bar{w}_{u, i}\right\|_{0, \infty, \tau_{j}} \lesssim h^{k+2 i+1}\|u\|_{k+1+2 i, \infty, \tau_{j}} \\
\left\|w_{u, i}\right\|_{0, \infty, \tau_{j}} & \lesssim h^{k+2 i}\|u\|_{k+2 i, \infty, \tau_{j}}, \quad\left\|\bar{w}_{q, i}\right\|_{0, \infty, \tau_{j}} \lesssim h^{k+2 i+1}\|u\|_{k+2+2 i, \infty, \tau_{j}}
\end{aligned}
$$

利用校正函数, 我们可得到如下的超逼近结果.

引理 $3.2^{[23]}$ 令 $u \in W^{k+l+3, \infty}(\Omega), 1 \leqslant l \leqslant k$ 是方程 (3.1) 的解, 则对流量 (3.4) 和 (3.5) 均有

$$
\left|a^{1}\left(u-u_{I}^{l}, q-q_{I}^{l} ; v\right)\right|+\left|a^{2}\left(u-u_{I}^{l}, q-q_{I}^{l} ; \varphi\right)\right| \lesssim h^{k+l+1}\|u\|_{k+l+3, \infty}\left(\|v\|_{0,1}+\|\varphi\|_{0,1}\right), \quad \forall v, \varphi \in V_{h} .
$$

此外, 若 $\left(u_{h}, q_{h}\right)$ 是方程 $(3.3)$ 的解且初值满足 $u_{h}(x, 0)=u_{I}^{l}(x, 0)$, 那么,

$$
\left\|u_{I}^{l}-u_{h}\right\|_{0}+\left\|q_{I}^{l}-q_{h}\right\|_{0} \lesssim(1+t) h^{k+l+1}\|u\|_{k+l+3, \infty} .
$$

\section{3 超收敛}

利用 $\left(u_{I}^{l}, q_{I}^{l}\right)$ 和数值解 $\left(u_{h}, q_{h}\right)$ 的超逼近结果, 接下来研究 $\left(u_{h}, q_{h}\right)$ 的超收玫性质. 同样地, 我们 将改进文献 [23] 中的超收玫结果, 把节点上的逐点误差收玫阶由原来的 $h^{2 k+\frac{1}{2}}$ 提高到 $h^{2 k+1}$, 而区间 平均值误差收玫阶则从原来的 $h^{2 k}$ 提高到 $h^{2 k+1}$.

定理 3.3 设 $u \in W^{2 k+3, \infty}(\Omega)$ 和 $\left(u_{h}, q_{h}\right)$ 分别是方程 (3.1) 和 (3.3) 的解. 若初值 $u_{h}(\cdot, 0)=$ $u_{I}^{k}(x, 0)$, 则对周期和混合边界条件均有

$$
\left\|u-u_{h}\right\|_{c}+\left\|q-q_{h}\right\|_{c} \lesssim(1+t) h^{2 k+1}\|u\|_{2 k+3, \infty} .
$$

证明 利用 $P_{h}^{-}$和 $P_{h}^{+}$的性质以及正交性质 (3.10) 可得, 当 $k=2 r+1$ 为奇数时,

$$
\int_{\tau_{j}}\left(u-u_{h}\right)=\int_{\tau_{j}}\left(u_{I}^{k}-u_{h}\right)+\int_{\tau_{j}} w_{u, r+1}
$$

当 $k=2 r$ 为偶数时,

$$
\int_{\tau_{j}}\left(u-u_{h}\right)=\int_{\tau_{j}}\left(u_{I}^{k}-u_{h}\right)+\int_{\tau_{j}} \bar{w}_{u, r} .
$$


在 (3.13) 中取 $l=k$ 并结合不等式 (3.11) 和 (3.12) 即可得

$$
\left\|u-u_{h}\right\|_{c} \lesssim\left\|u_{I}^{k}-u_{h}\right\|_{0}+h^{2 k+1}\|u\|_{2 k+3, \infty} \lesssim(1+t) h^{2 k+1}\|u\|_{2 k+3, \infty} .
$$

同理, 我们可证明相同的结果对 $\left\|q-q_{h}\right\|_{c}$ 也成立. 从而, 定理得证.

与守恒律方程相同, 在分析 $\left(u_{h}, q_{h}\right)$ 的数值迹在节点的超收玫性质之前, 我们也需要估计 $\|\left(u_{I}^{k}\right.$ $\left.-u_{h}\right)_{t} \|_{0}$. 为简单起见, 我们记

$$
\left(e_{u}, e_{q}\right)=\left(u-u_{h}, q-q_{h}\right), \quad\left(\xi_{u}, \xi_{q}\right)=\left(u_{I}^{k}-u_{h}, q_{I}^{k}-q_{h}\right) .
$$

引理 3.4 设 $u \in W^{2 k+5, \infty}(\Omega)$ 和 $\left(u_{h}, q_{h}\right)$ 分别是方程 (3.1) 和 (3.3) 的解. 若初值 $u_{h}(\cdot, 0)=$ $u_{I}^{k}(x, 0)$, 则对周期和混合边界条件均有

$$
\left\|\left(u_{I}^{k}-u_{h}\right)_{t}\right\|_{0} \lesssim(1+t) h^{2 k+1}\|u\|_{2 k+5, \infty} .
$$

证明 对于三种不同的边界条件, 根据我们对边界数值流量的选择和校正函数的构造可得

$$
\left.\left(\hat{u}_{I}^{k}-\hat{u}_{h}\right)_{t}\left(\hat{q}-\hat{q}_{h}\right)_{t}\right|_{N+\frac{1}{2}}=\left.\left(\hat{u}_{I}^{k}-\hat{u}_{h}\right)_{t}\left(\hat{q}-\hat{q}_{h}\right)_{t}\right|_{\frac{1}{2}} .
$$

从而, 在 (3.6) 或者 $(3.7)$ 中取 $(\varepsilon, \eta)=\left(\xi_{u t}, \xi_{q t}\right)$ 并利用上述等式和正交性质

$$
a^{1}\left(e_{u t}, e_{q t} ; v\right)+a^{2}\left(e_{u t}, e_{q t} ; \varphi\right)=0, \quad \forall v, \varphi \in V_{h},
$$

我们可得

$$
\begin{aligned}
\frac{1}{2} \frac{d}{d t}\left(\xi_{u t}, \xi_{u t}\right)+\left(\xi_{q t}, \xi_{q t}\right) & =a^{1}\left(\xi_{u t}, \xi_{q t} ; \xi_{u t}\right)+a^{2}\left(\xi_{u t}, \xi_{q t} ; \xi_{q t}\right) \\
& =-a^{1}\left(\left(u-u_{I}^{k}\right)_{t},\left(q-q_{I}^{k}\right)_{t} ; \xi_{u t}\right)-a^{2}\left(\left(u-u_{I}^{k}\right)_{t},\left(q-q_{I}^{k}\right)_{t} ; \xi_{q t}\right) .
\end{aligned}
$$

由 (3.8) 和 (3.9) 可得, 当 $k=2 r$ 为偶数时,

$$
a^{1}\left(\left(u-u_{I}^{k}\right)_{t},\left(q-q_{I}^{k}\right)_{t} ; \xi_{u t}\right)=\left(\left(\bar{w}_{u, r}\right)_{t t}, \xi_{u t}\right), \quad a^{2}\left(\left(u-u_{I}^{k}\right)_{t},\left(q-q_{I}^{k}\right)_{t} ; \xi_{q t}\right)=\left(\left(\bar{w}_{q, r}\right)_{t}, \xi_{q t}\right) ;
$$

当 $k=2 r+1$ 为奇数时,

$$
a^{1}\left(\left(u-u_{I}^{k}\right)_{t},\left(q-q_{I}^{k}\right)_{t} ; \xi_{u t}\right)=\left(\left(w_{u, r+1}\right)_{t t}, \xi_{u t}\right), \quad a^{2}\left(\left(u-u_{I}^{k}\right)_{t},\left(q-q_{I}^{k}\right)_{t} ; \xi_{q t}\right)=\left(\left(w_{q, r+1}\right)_{t}, \xi_{q t}\right) .
$$

鉴于估计式 (3.11)、(3.12) 和 $u_{t}=-u_{x x}$, 对任意的 $k \geqslant 1$,

$$
\left|a^{1}\left(\left(u-u_{I}^{k}\right)_{t},\left(q-q_{I}^{k}\right)_{t} ; \xi_{u t}\right)+a^{2}\left(\left(u-u_{I}^{k}\right)_{t},\left(q-q_{I}^{k}\right)_{t} ; \xi_{q t}\right)\right| \lesssim h^{2 k+1}\|u\|_{2 k+5, \infty}\left(\left\|\xi_{u t}\right\|_{0}+\left\|\xi_{q t}\right\|_{0}\right),
$$

从而,

$$
\frac{d}{d t}\left\|\xi_{u t}\right\|_{0}^{2}+\left\|\xi_{q t}\right\|_{0}^{2} \lesssim h^{2 k+1}\|u\|_{2 k+5, \infty}\left(\left\|\xi_{u t}\right\|_{0}+\left\|\xi_{q t}\right\|_{0}\right) .
$$

对上式运用 Cauchy-Schwarz 不等式可得

$$
\frac{d}{d t}\left\|\xi_{u t}\right\|_{0}^{2} \lesssim h^{2 k+1}\|u\|_{2 k+5, \infty}\left(\left\|\xi_{u t}\right\|_{0}+h^{2 k+1}\|u\|_{2 k+5, \infty}\right) .
$$

从而, 由 Gronwall 不等式可推导出

$$
\left\|\xi_{u t}\right\|_{0}(t) \lesssim\left\|\xi_{u t}\right\|_{0}(0)+t h^{2 k+1}\|u\|_{2 k+5, \infty}, \quad \forall t>0 .
$$


为了证明 (2.10), 接下来只需分析 $\left\|\xi_{u t}\right\|_{0}(0)$ 即可. 在 $t=0$ 时刻, 因为 $\xi_{u}=0$, 从而, 由间断有限元格 式可得

$$
\begin{aligned}
0=a^{2}\left(e_{u}, q_{u} ; v\right)(0) & =a^{2}\left(\xi_{u}, \xi_{q} ; v\right)(0)+a^{2}\left(u-u_{I}^{k}, q-q_{I}^{k} ; v\right)(0) \\
& =\left(\xi_{q}, v\right)(0)+a^{2}\left(u-u_{I}^{k}, q-q_{I}^{k} ; v\right)(0) .
\end{aligned}
$$

由 (3.8) 和 (3.9), 我们不难得到

$$
\xi_{q}(x, 0)=-\bar{w}_{q, r}(x, 0), \quad \text { 若 } k=2 r, \quad \xi_{q}(x, 0)=-w_{q, r+1}(x, 0), \quad \text { 若 } k=2 r+1 .
$$

从而, 由 (3.11) 和 (3.12) 可得

$$
\left\|\partial_{t}^{m} \xi_{q}\right\|_{0, \infty}(0) \lesssim h^{2 k+1}\left\|\partial_{t}^{m} u\right\|_{2 k+3, \infty}, \quad m=0,1 .
$$

另一方面, 不妨设 $\xi_{u t}(x, 0)$ 在区间 $\tau_{j}, j \in \mathbb{Z}_{N}$ 上有如下表达式:

$$
\xi_{u t}(x, 0)=\xi_{u t}\left(x_{j}\right)+s(x) \frac{x-x_{j}}{h_{j}}, \quad x \in \tau_{j} .
$$

若 $\hat{u}_{h}=u_{h}^{-}$, 则在方程 $a_{j}^{2}\left(e_{u t}, e_{q t} ; v\right)=0$ 取 $v=s(x) \frac{x-x_{j-\frac{1}{2}}}{h_{j}}$ 并按照定理 2.4 同样的证明方法可推出

$$
\|s\|_{0, \tau_{j}}^{2} \lesssim h_{j}\left|a_{j}^{2}\left(\left(u-u_{I}^{k}\right)_{t},\left(q-q_{I}^{k}\right)_{t} ; v\right)(0)+\left(\xi_{q t}, v\right)_{j}(0)\right| \lesssim h^{2 k+2}\|u\|_{2 k+5, \infty}\|v\|_{0,1, \tau_{j}}
$$

利用 $\|v\|_{0,1, \tau_{j}} \lesssim h^{\frac{1}{2}}\|s\|_{0, \tau_{j}}$ 和逆不等式, 我们可得

$$
\|s\|_{0, \tau_{j}} \lesssim h^{2 k+2+\frac{1}{2}}\|u\|_{2 k+5, \infty}, \quad\|s\|_{0, \infty, \tau_{j}} \lesssim h_{j}^{-\frac{1}{2}}\|s\|_{0, \tau_{j}} \lesssim h^{2 k+2}\|u\|_{2 k+5, \infty} .
$$

在方程 $a_{j}^{2}\left(e_{u t}, e_{q t} ; v\right)=0$ 中取 $v=1$ 可得

$$
\left.\xi_{u t}^{-}\right|_{j+\frac{1}{2}}-\left.\xi_{u t}^{-}\right|_{j-\frac{1}{2}}=\left.e_{u t}^{-}\right|_{j+\frac{1}{2}}-\left.e_{u t}^{-}\right|_{j-\frac{1}{2}}=\int_{\tau_{j}} e_{q t}=\int_{\tau_{j}}\left(\xi_{q}+w_{q}^{k}\right)_{t} .
$$

从而,

$$
\left.\xi_{u t}^{-}\right|_{j+\frac{1}{2}}=\left.\xi_{u t}^{-}\right|_{\frac{1}{2}}+\sum_{i=1}^{j} \int_{\tau_{i}}\left(\xi_{q}+w_{q}^{k}\right)_{t} .
$$

在 $t=0$ 时刻把 $\xi_{u t}$ 的表达式代入上式可得

$$
\xi_{u t}\left(x_{j}\right)=\xi_{u t}\left(x_{1}\right)+\frac{1}{2} s\left(x_{\frac{1}{2}}^{-}\right)-\frac{1}{2} s\left(x_{j+\frac{1}{2}}^{-}\right)+\sum_{i=1}^{j} \int_{\tau_{i}}\left(\xi_{q}+w_{q}^{k}\right)_{t}(0) .
$$

同理, 在方程 $a^{1}\left(e_{u}, e_{q} ; v\right)=0$ 中取 $v=1$,

$$
\sum_{j=1}^{N} \int_{\tau_{j}}\left(\xi_{u}+w_{u}^{k}\right)_{t}=\sum_{j=1}^{N} \int_{\tau_{j}} e_{u t}=\left.e_{q}^{+}\right|_{N+\frac{1}{2}}-\left.e_{q}^{+}\right|_{\frac{1}{2}} .
$$

在上式中取 $t=0$ 并把 $\xi_{u t}$ 的表达式代入可得

$$
\sum_{j=1}^{N}\left(h_{j} \xi_{u t}\left(x_{j}\right)+\int_{\tau_{j}} s(x) \frac{x-x_{j}}{h_{j}}\right)=-\sum_{j=1}^{N} \int_{\tau_{j}}\left(w_{u}^{k}\right)_{t}(0)+\xi_{q}\left(x_{N+\frac{1}{2}}^{+}, 0\right)-\xi_{q}\left(x_{\frac{1}{2}}^{+}, 0\right) .
$$


把 (3.17) 代入上式并利用 $\|s\|_{0, \infty}$ 和 $\left\|\partial_{t}^{m} \xi_{q}\right\|_{0, \infty}(0), m=0,1$ 的估计式可得

$$
\begin{aligned}
\left|\xi_{u t}\left(x_{1}\right)\right| & \lesssim\|s\|_{0, \infty}+\sum_{m=0}^{1}\left\|\partial_{t}^{m} \xi_{q}\right\|_{0, \infty}(0)+\left|\sum_{j=1}^{N} \int_{\tau_{j}}\left(w_{u}^{k}\right)_{t}(0)\right|+\left|\sum_{j=1}^{N} \int_{\tau_{j}}\left(w_{q}^{k}\right)_{t}(0)\right| \\
& \lesssim h^{2 k+1}\|u\|_{2 k+5, \infty}
\end{aligned}
$$

其中最后一步用到了估计式 (3.11)、(3.12) 和

$$
\int_{\tau_{j}} w_{v t}=\int_{\tau_{j}}\left(\bar{w}_{v, r}\right)_{t}, \quad \text { 若 } k=2 r, \quad \int_{\tau_{j}} w_{v t}=\int_{\tau_{j}}\left(w_{v, r+1}\right)_{t}, \quad \text { 若 } k=2 r+1, \quad v=u, q .
$$

从而, 对任意的 $j \in \mathbb{Z}_{N}$, 我们从 (3.17) 推导出

$$
\left|\xi_{u t}\left(x_{j}\right)\right| \lesssim\left|\xi_{u t}\left(x_{1}\right)\right|+\sum_{i=1}^{j}\left|\int_{\tau_{i}}\left(w_{q}^{k}\right)_{t}(0)\right|+\left\|\xi_{q t}\right\|_{0, \infty}(0)+\|s\|_{0, \infty} \lesssim h^{2 k+1}\|u\|_{2 k+5, \infty} .
$$

把 $\xi_{u t}\left(x_{j}\right)$ 和 $s(x)$ 的估计式联立起来即可得

$$
\left\|\xi_{u t}\right\|_{0}(0) \lesssim\left\|\xi_{u t}\right\|_{0, \infty}(0) \lesssim h^{2 k+1}\|u\|_{2 k+5, \infty}
$$

从而, 对任意的 $t>0$,

$$
\left\|\xi_{u t}\right\|_{0} \lesssim(1+t) h^{2 k+1}\|u\|_{2 k+5, \infty},
$$

即当流量取为 $(3.4)$ 时, 不等式 (3.15) 成立. 若 $\hat{u}_{h}=u_{h}^{+}$, 即流量取 $(3.5)$ 时, 在方程 $a_{j}^{2}\left(e_{u t}, e_{q t} ; v\right)=0$ 中取 $v=s(x) \frac{x-x_{j+\frac{1}{2}}}{h_{j}}$ 并利用等式 (参见文献 [24])

$$
\int_{\tau_{j}} s(x) \frac{x-x_{j+\frac{1}{2}}}{h_{j}} \frac{d}{d x}\left(s(x) \frac{x-x_{j}}{h_{j}}\right)=-\frac{1}{4 h_{j}} \int_{\tau_{j}} s^{2}(x)-\frac{s^{2}\left(x_{j-\frac{1}{2}}\right)}{4},
$$

我们同样可证得 (3.16). 按照与前面类似的证明方法, 我们可证明 (3.15) 在流量取 (3.5) 时仍成立. 从 而, 结论得证.

定理 3.5 设 $u \in W^{2 k+5, \infty}(\Omega)$ 和 $\left(u_{h}, q_{h}\right)$ 分别是方程 (3.1) 和 (3.3) 的解. 若初始时刻 $u_{h}(\cdot, 0)=$ $u_{I}^{k}(x, 0)$, 则对周期和混合边界条件均有

$$
\left|\left(u-\hat{u}_{h}\right)\left(x_{j-\frac{1}{2}}, t\right)\right|+\left|\left(q-\hat{q}_{h}\right)\left(x_{j-\frac{1}{2}}, t\right)\right| \lesssim(1+t) h^{2 k+1}\|u\|_{2 k+5, \infty}, \quad \forall j \in \mathbb{Z}_{N+1} .
$$

证明回顾间断有限元格式, 我们可得

$$
\begin{aligned}
& \left.\hat{\xi}_{u}\right|_{j+\frac{1}{2}}-\left.\hat{\xi}_{u}\right|_{j-\frac{1}{2}}=\left.\hat{e}_{u}\right|_{j+\frac{1}{2}}-\left.\hat{e}_{u}\right|_{j-\frac{1}{2}}=\int_{\tau_{j}} e_{q}=\int_{\tau_{j}}\left(\xi_{q}+w_{q}^{k}\right), \\
& \left.\hat{\xi}_{q}\right|_{j+\frac{1}{2}}-\left.\hat{\xi}_{q}\right|_{j-\frac{1}{2}}=\left.\hat{e}_{q}\right|_{j+\frac{1}{2}}-\left.\hat{e}_{q}\right|_{j-\frac{1}{2}}=\int_{\tau_{j}} e_{u t}=\int_{\tau_{j}}\left(\xi_{u}+w_{u}^{k}\right)_{t} .
\end{aligned}
$$

由 (3.10) 可得

$$
\left.\hat{\xi}_{u}\right|_{j+\frac{1}{2}}-\left.\hat{\xi}_{u}\right|_{\frac{1}{2}}=\sum_{i=1}^{j} \int_{\tau_{i}}\left(\xi_{q}+w_{q, r+1}\right),\left.\quad \hat{\xi}_{q}\right|_{j+\frac{1}{2}}-\left.\hat{\xi}_{q}\right|_{\frac{1}{2}}=\sum_{i=1}^{j} \int_{\tau_{i}}\left(\xi_{u}+w_{u, r+1}\right)_{t}, \quad \text { 当 } k=2 r+1,
$$




$$
\left.\hat{\xi}_{u}\right|_{j+\frac{1}{2}}-\left.\hat{\xi}_{u}\right|_{\frac{1}{2}}=\sum_{i=1}^{j} \int_{\tau_{i}}\left(\xi_{q}+\bar{w}_{q, r}\right),\left.\quad \hat{\xi}_{q}\right|_{j+\frac{1}{2}}-\left.\hat{\xi}_{q}\right|_{\frac{1}{2}}=\sum_{i=1}^{j} \int_{\tau_{i}}\left(\xi_{u}+\bar{w}_{u, r}\right)_{t}, \quad \text { 当 } k=2 r .
$$

当边界条件为 $u(0, t)=g_{0}(t), u_{x}(2 \pi, t)=g_{1}(t)$, 流量取 (3.4) 时, 因为

$$
\left.\hat{e}_{u}\right|_{\frac{1}{2}}=\left.e_{u}^{-}\right|_{\frac{1}{2}}=\left.\xi_{u}^{-}\right|_{\frac{1}{2}}=0,\left.\quad \hat{e}_{q}\right|_{N+\frac{1}{2}}=\left.e_{q}^{+}\right|_{N+\frac{1}{2}}=\left.\xi_{q}^{+}\right|_{N+\frac{1}{2}}=0,
$$

所以, 从 (3.15)、(3.11) 和 (3.12), 我们可推导出

$$
\left|\left(u-u_{h}\right)\left(x_{j-\frac{1}{2}}^{-}, t\right)\right|+\left|\left(q-q_{h}\right)\left(x_{j-\frac{1}{2}}^{+}, t\right)\right| \lesssim(1+t) h^{2 k+1}\|u\|_{2 k+5, \infty}, \quad \forall j \in \mathbb{Z}_{N+1} .
$$

同样地, 当边界条件为 $u_{x}(0, t)=g_{0}(t), u(2 \pi, t)=g_{1}(t)$, 流量取 (3.5) 时, 因为

$$
\left.\hat{e}_{q}\right|_{\frac{1}{2}}=\left.e_{q}^{-}\right|_{\frac{1}{2}}=\left.\xi_{q}^{-}\right|_{\frac{1}{2}}=0,\left.\quad \hat{e}_{u}\right|_{N+\frac{1}{2}}=\left.e_{u}^{+}\right|_{N+\frac{1}{2}}=\left.\xi_{u}^{+}\right|_{N+\frac{1}{2}}=0,
$$

从而, 对任意的 $j \in \mathbb{Z}_{N+1}$,

$$
\left|\left(u-u_{h}\right)\left(x_{j-\frac{1}{2}}^{+}, t\right)\right|+\left|\left(q-q_{h}\right)\left(x_{j-\frac{1}{2}}^{-}, t\right)\right| \lesssim(1+t) h^{2 k+1}\|u\|_{2 k+5, \infty} .
$$

接下来考虑周期边界条件. 在任意的区间 $\tau_{j}, j \in \mathbb{Z}_{N}$ 中, 不妨设

$$
\xi_{u}(x, t)=\xi_{u}\left(x_{j}, t\right)+s_{u}(x, t) \frac{x-x_{j}}{h_{j}}, \quad \forall x \in \tau_{j} .
$$

按照引理 3.4 同样的证明方法, 我们不难得到

$$
\left\|s_{u}\right\|_{0, \tau_{j}}^{2} \lesssim h_{j}\left|\left(\xi_{q}, v\right)_{j}+a_{j}^{2}\left(u-u_{I}^{k}, q-q_{I}^{k} ; v\right)\right| \lesssim\left(h^{2 k+2}\|u\|_{2 k+5, \infty}+\left\|\xi_{q}\right\|_{0}\right)\|v\|_{0, \tau_{j}},
$$

其中

$$
v=s_{u} \frac{x-x_{j-\frac{1}{2}}}{h_{j}} \text {, 当 }\left(\hat{u_{h}}, \hat{q_{h}}\right)=\left(u_{h}^{-}, q_{h}^{+}\right), \quad v=s_{u} \frac{x-x_{j+\frac{1}{2}}}{h_{j}} \text {, 当 }\left(\hat{u_{h}}, \hat{q_{h}}\right)=\left(u_{h}^{+}, q_{h}^{-}\right) \text {. }
$$

从而, 由逆不等式和 (3.13) 可得

$$
\left\|s_{u}\right\|_{0, \infty, \tau_{j}} \lesssim h_{j}^{-\frac{1}{2}}\left\|s_{u}\right\|_{0, \tau_{j}} \lesssim(1+t) h^{2 k+\frac{3}{2}}\|u\|_{2 k+5, \infty} .
$$

同理, 按照引理 3.4 类似的证明思路, 我们可证得对任意的 $t>0$ 均有

$$
\left|\xi_{u}\left(x_{j}, t\right)\right| \lesssim(1+t) h^{2 k+1}\|u\|_{2 k+5, \infty}
$$

从而,

$$
\left|\hat{e}_{u}\left(x_{j+\frac{1}{2}}, t\right)\right|=\left|\hat{\xi}_{u}\left(x_{j+\frac{1}{2}}, t\right)\right| \lesssim\left\|\xi_{u}\right\|_{0, \infty} \lesssim(1+t) h^{2 k+1}\|u\|_{2 k+5, \infty} .
$$

用同样的方法我们可证明

$$
\left|\hat{e}_{q}\left(x_{j+\frac{1}{2}}, t\right)\right| \lesssim(1+t) h^{2 k+1}\|u\|_{2 k+5, \infty} .
$$

所以, 无论是选择流量 (3.4) 还是 (3.5), (3.18) 对于周期边界条件都成立. 定理得证.

注 3.6 与守恒律方程相同, 为了证明数值解在节点上的逐点误差以 $2 k+1$ 的速度超收玫, 我们 对解的正则性要求 $W^{2 k+5, \infty}(\Omega)$ 高于文献 [23] 所要求的正则性条件 $W^{2 k+3, \infty}(\Omega)$. 


\section{参考文献}

1 Reed W H, Hill T R. Triangular Mesh for Neutron Transport Equation. Los Alamos Scientific Laboratory Report LA-UR-73-479. Los Alamos, NM: Petley Studios Inc, 1973

2 Cockburn B, Hou S, Shu C W. The Runge-Kutta local projection discontinuous Galerkin finite element method for conservation laws, IV: The multidimensional case. Math Comp, 1990, 54: 545-581

3 Cockburn B, Lin S, Shu C W. TVB Runge-Kutta local projection discontinuous Galerkin finite element method for conservation laws, III: One dimensioal systems. J Comput Phys, 1989, 84: 90-113

4 Cockburn B, Shu C W. TVB Runge-Kutta local projection discontinuous Galerkin finite element method for coservation laws, II: Genaral framework. Math Comp, 1989, 52: 411-435

5 Cockburn B, Shu C W. The Runge-Kutta local projection P1-discontinuous Galerkin finite element method for scalar coservation laws. RAIRO Modél Math Anal Numér, 1991, 25: 337-361

6 Cockburn B, Shu C W. The Runge-Kutta discontinuous Galerkin method for conservation laws, V: Multidimensional systems. J Comput Phys, 1998, 141: 199-224

7 Cockburn B, Shu C W. The local discontinuous Galerkin method for time-dependent convection-diffusion systems. SIAM J Numer Anal, 1998, 35: 2440-2463

8 Yan J, Shu C W. A local discontinuous Galerkin methods for Kdv type equations. SIAM J Numer Anal, 2002, 40: 769-791

9 Xia Y, Xu Y, Shu C W. Local discontinuous Galerkin methods for the Cahn-Hilliard type equations. J Comput Phys, 2007, 227: 472-491

$10 \mathrm{Xu}$ Y, Shu C W. Local discontinuous Galerkin methods for three classes of nonlinear wave equations. J Comput Math, 2004, 22: 250-274

$11 \mathrm{Xu} \mathrm{Y,} \mathrm{Shu} \mathrm{C} \mathrm{W.} \mathrm{Local} \mathrm{discontinuous} \mathrm{Galerkin} \mathrm{methods} \mathrm{for} \mathrm{Kuramoto-Sivashinsky} \mathrm{equations} \mathrm{and} \mathrm{the} \mathrm{Itô-type} \mathrm{coupled}$ Kdv equations. Comuput Methods Appl Mech Engrg, 2006, 195: 3430-3447

12 Yan J, Shu C W. Local discontinuous Galerkin methods for partial differential equations with higher derivatives. J Sci Comput, 2002, 17: 27-47

13 Adjerid S, Devine K D, Flaherty J E, et al. A posteriori error estimation for discontinuous Galerkin solutions of hyperbolic problems. Comput Methods Appl Mech Engrg, 2002, 191: 1097-1112

14 Adjerid S, Weinhart T. Discontinuous Galerkin error estimation for linear symmetrizable hyperbolic systems. Math Comp, 2011, 80: 1335-1367

15 Celiker F, Cockburn B. Superconvergence of the numerical traces of discontinuous Galerkin and hybridized methods for convection-diffusion problems in one space dimension. Math Comp, 2007, 76: 67-96

16 Cheng Y, Shu C W. Superconvergence and time evolution of discontinuous Galerkin finite element solutions. J Comput Phys, 2008, 227: 9612-9627

17 Guo W, Zhong X, Qiu J. Superconvergence of discontinuous Galerkin and local discontinuous Galerkin methods: Eigen-structure analysis based on Fourier approach. J Comput Phys, 2013, 235: 458-485

18 林群. 中子迁移方程的不连续有限元的外推. 控制理论与应用, 1999, 16: 20-22

19 Xie Z, Zhang Z. Uniform superconvergence analysis of the discontinuous Galerkin method for a singularly perturbed problem in 1-D. Math Comp, 2010, 79: 35-45

20 Zhang Z, Xie Z, Zhang Z. Superconvergence of discontinuous Galerkin methods for convection-diffusion problems. J Sci Comput, 2009, 41: 70-93

21 Yang Y, Shu C W. Analysis of optimal supercovergence of discontinuous Galerkin method for linear hyperbolic equations. SIAM J Numer Anal, 2012, 50: 3110-3133

22 Cao W, Zhang Z, Zou Q. Superconvergence of discontinuous Galerkin method for linear hyperbolic equations. SIAM J Numer Anal, 2014, 52: 2555-2573

23 Cao W, Zhang Z. Superconvergence of local discontinuous Galerkin method for one-dimensional linear parabolic equations. ArXiv:1401.5150v1, 2014

24 Cheng Y, Shu C W. Superconvergence of discontinuous Galerkin and local discontinuous Galerkin schemes for linear hyperbolic and convection-diffusion equations in one space dimension. SIAM J Numer Anal, 2010, 47: 4044-4072

25 陈传永. 有限元超收玫构造理论. 长沙: 湖南科技出版社, 2001

26 陈传沝, 黄云清. 有限元高精度理论. 长沙: 湖南科技出版社, 1995

27 Lin Q, Lin J. Finite Element Methods: Accuracy and Improvement. Beijing: Science Press, 2006

28 林群, 严宁宁. 高效有限元构造与分析. 保定: 河北大学出版社, 1996

29 Wahlbin L R. Superconvergence in Galerkin Finite Element Methods. Lecture Notes in Mathematics, vol. 1605. Berlin: Springer, 1995 
30 朱起定, 林群. 有限元超收玫理论. 长沙: 湖南科技出版社, 1989

\title{
Point-wise and cell average error estimates of the DG and LDG methods for 1D hyperbolic and parabolic equations
}

\section{CAO WaiXiang \& ZHANG ZhiMin}

\begin{abstract}
In this paper, we study superconvergence properties of the discontinuous and local discontinuous Galerkin methods for 1D hyperbolic conservation laws and parabolic equations. Specifically, when using upwind fluxes (for hyperbolic conservation laws) and alternating fluxes (for parabolic equations), we prove for any polynomial degree $k$, a $(2 k+1)$-th superconvergence rate for the error of cell average, as well as the point-wise error estimates of the DG approximation at downwind points (hyperbolic) or the LDG approximation of numerical traces at nodes (parabolic). These superconvergence results are completely consistent with the numerical results provided in Cao et al. (2014), Cao and Zhang (2014) and Yang and Shu (2012), and improve the theoretical results in Cao et al. (2014) and Cao and Zhang (2014), which is $\frac{1}{2}$ order and one order higher than that in Cao et al. (2014) and Cao and Zhang (2014) for the point-wise error estimates and cell average, respectively.
\end{abstract}

Keywords superconvergence, discontinuous Galerkin method, cell average, hyperbolic equations, parabolic equations, point-wise error estimate

$\operatorname{MSC}(2010) \quad 65 \mathrm{M} 15,65 \mathrm{M} 60,65 \mathrm{~N} 30$

doi: $10.1360 /$ N012014-00214 